\title{
PREOCUPACIÓN ASOCIADA AL DESCUBRIMIENTO DE LA ORIENTACIÓN SEXUAL
}

\author{
Naiara Martinez-Gomez \\ Grupo Salusex. Departamento de Psicologia Basica, Clinica y Psicobiologia \\ Universitat Jaume I de Castellon \\ gomezn@uji.es \\ Rafael Ballester-Arnal \\ Grupo Salusex. Departamento de Psicologia Basica, Clinica y Psicobiologia \\ Universitat Jaume I de Castellon \\ Cristina Gimenez-Garcia \\ Grupo Salusex. Departamento de Psicologia Basica, Clinica y Psicobiologia \\ Universitat Jaume I de Castellon \\ Estefanía Ruiz-Palomino \\ Grupo Salusex. Departamento de Psicologia Basica, Clinica y Psicobiologia \\ Universitat Jaume I de Castellon \\ Juan Enrique Nebot-Garcia \\ Grupo Salusex. Departamento de Psicologia Basica, Clinica y Psicobiologia \\ Universitat Jaume I de Castellon
}

Recepción Artículo: 15 enero 2020

Admisión Evaluación: 4 marzo 2020

Informe Evaluador 1: 17 marzo 2020

Informe Evaluador 2: 15 marzo 2020

Aprobación Publicación: 20 abril 2020

Este trabajo ha sido posible en parte gracias a la ayuda de la Universitat Jaume I para el proyecto de investigación UJI-B2018-42

\section{RESUMEN}

El nivel de aceptación por parte de la sociedad sobre la diversidad afectivo-sexual influye directamente en la vivencia de las personas LGTBI. El hecho de tener que ocultar la propia orientación sexual o vivirla con elevada preocupación fomenta el desarrollo de homofobia interiorizada, con los consiguientes problemas de salud mental que ésta produce. Nuestro objetivo fue analizar las diferencias existentes entre adolescentes y población joven en relación a la vivencia y la preocupación por la propia orientación sexual. Para ello contamos con una muestra de 140 adolescentes y jóvenes (65\% mujeres y 35\% hombres) entre 12 y 25 años, de dos centros públicos de educación secundaria de la provincia de Castellón. Los participantes cumplimentaron un cuestionario on-line desarrollado ad hoc de 52 ítems. Para este estudio hemos seleccionado los 3 ítems que evalúan la vivencia y la preocupación por la orientación sexual. Ya entre los 12 y 18 años, un 1.1\% de adolescentes se siente bisexual, un $3 \%$ homosexual, un $4 \%$ tiene dudas y un $14 \%$ no siente atracción sexual por ningún sexo. Las personas no heterosexuales mostraron mayor preocupación al darse cuenta de su orientación que las personas heterosexuales (un 


\section{PREOCUPACIÓN ASOCIADA AL DESCUBRIMIENTO DE LA ORIENTACIÓN SEXUAL}

45.5\% frente a un $3.4 \%)$ siendo estas diferencias significativas estadísticamente $\left(\chi^{2}=40.505 ; \mathrm{p} £ .001\right)$. Al analizar la preocupación por su orientación en la actualidad, en el caso de heterosexuales se mantiene en un 3.5\% frente a un $31.8 \%$ de no heterosexuales $\left(\chi^{2}=23.838 ; p £ .001\right)$. Aunque las diferencias no resultaron estadísticamente significativas $\left(\chi^{2}=.404 ; p=.525\right)$, observamos que los adolescentes (12-18 años) no heterosexuales presentan menos preocupación que los jóvenes no heterosexuales de entre 19 a 25 años (75\% frente a un 61.5\%). En cuanto a la edad en que se dieron cuenta de su orientación, en las personas no heterosexuales fue posterior $(M=10.45 ; D T=6.43)$ que en las heterosexuales ( $M=5.74 ; D T=5.04)$ siendo estas diferencias estadísticamente significativas $(t=3.84 ; £ .001)$. Finalmente, las personas no heterosexuales, cuanto más tarde se dieron cuenta de su orientación sexual, más preocupación mostraban en la actualidad. Estos resultados muestran que todavía jóvenes y adolescentes presentan preocupación sobre su propia orientación sexual, sobre todo cuando la identificación como no heterosexual se hace a una edad más tardía. Resaltamos la importancia de normalizar la diversidad afectivo-sexual, y la necesidad de romper con la heteronormatividad, para que identificarse como no heterosexual no implique ningún tipo de preocupación que puede ser la antesala de problemas de salud mental.

Palabras clave: orientación sexual; LGTBI; preocupación; homofobia interiorizada; toma de conciencia

\section{ABSTRACT}

Concern associated with discovering own's sexual orientation. The degree of social acceptance on sexual-affective diversity directly influences LGTBI individuals' lives. Hiding their sexual orientation or living with concerns lead to the development of internalized homophobia and, consequently, this may cause some mental health disorders. The aim was to analyze the differences between adolescents and young population in relation to their experience and concern on their sexual orientation. The sample was composed by 140 adolescents and young people ( $65 \%$ women and $35 \%$ men), ages ranging from 12 to 25 years old, belonging to two public secondary schools in the province of Castellón. Participants completed an ad hoc on-line questionnaire with 52 items. Three items were selected to assess participants' experience and concern on their sexual orientation. An $1.1 \%$ of adolescents aged 12 to 18 identified as bisexual, a 3\% as homosexual, a 4\% had doubts and a $14 \%$ did not feel sexual attraction to any sex. Non-heterosexual people showed greater concern when discovering their orientation than heterosexual people did (45.5\% vs. 3.4\%). There were statistically significant differences $\left(\chi^{2}=\right.$ 40.505; p£.001). At the present moment, heterosexual maintain their concern about orientation around $3.5 \%$ differing from non-heterosexuals who obtain $31.8 \%\left(\chi^{2}=23.838 ; p £ .001\right)$. In spite of the differences were not significant $\left(\chi^{2}=.404 ; p=.525\right)$, it was observed that non-heterosexual adolescents (12-18 years old) showed less concern than non-heterosexual young people aged $19-25$ (75\% vs. 61.5\%). Regarding the age at which they discovered their sexual orientation, results showed that non-heterosexual participants did it later ( $M=10.45$; $D T=$ 6.43) than heterosexual ones $(M=5.74 ; D T=5.04)$. There were statistically significant differences $(t=3.84$;

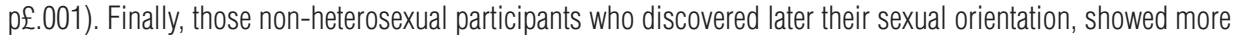
concern at present moment. These results have shown that young people and adolescents are still concerned on their own sexual orientation, especially in the cases that participants discovered a non-heterosexual one at a late age. This research emphasizes the importance of normalizing sexual-affective diversity and the need to break down hetero-normativity, so that identifying themselves as non-heterosexual individuals does not lead to any type of concern that may be the prelude to mental health disorders.

Keywords: sexual orientation; LGTBI; concern; internalized homophobia; awareness

\section{INTRODUCCIÓN}

De acuerdo con el modelo teórico propuesto por Cass (1979) y, más recientemente, adaptado por Soriano (2004), la construcción de la orientación sexual de las personas no heterosexuales pasa por un sentimiento de diferencia al resto de iguales seguido de un proceso de toma de conciencia de la propia orientación (una vez superada la etapa de negación y rechazo), que marcará el inicio de la autoidentificación. Todo este proceso se ve influenciado por el marco heteronormativo (Herz \& Johansson, 2015), que actúa como barrera dificultando el 
proceso. Uno de los factores que pueden influir en este proceso es la edad en la que la persona le haga frente. Pese a que no existen muchos trabajos que analicen este factor, un estudio llevado a cabo por Kertzner, Meyer, Frost y Stirratt (2009) defiende que cuanto menor es la edad, más se dificulta el proceso de empoderamiento de las personas que se encuentran fuera del marco heterosexual. Por ello, la adolescencia se trata de un periodo fundamental para establecer la orientación sexual de las personas dentro de este proceso identitario. En esta etapa vital encontramos que los iguales están todavía en proceso de exploración de su propia sexualidad. Este hecho conlleva que, en ocasiones, no encuentren entre sus pares personas visibles LGTBI (Lesbiana, Gay, Transexual, Bisexual e Intersexual) o estas se encuentren en fases de duda o cuestionamiento (Cornejo, 2012).

Así mismo, otro de los factores que influyen en el proceso identitario y en la normalización y visibilización de la vida afectivo-sexual es el nivel de aceptación por parte de la sociedad, que afecta directamente en la vivencia de las personas LGTBI. En los últimos años ha habido un avance en los derechos de personas pertenecientes al colectivo LGTBI con el establecimiento de leyes autonómicas y estatales, así como una mayor visibilización y normalización de las diferentes orientaciones sexuales e identidades de género (Asamblea General de la Asociación Mundial de Sexología, (1997; Montagut, 2015). No obstante, cabe recordar que la homosexualidad ha sido considerada una enfermedad hasta el año 1973 en el Manual diagnóstico y estadístico de los trastornos mentales (DSM) y hasta el año 1990 para la Organización Mundial de la Salud (OMS). El que durante tantos siglos, la homosexualidad haya sido considerada un pecado 0 un trastorno ha significado la consolidación de creencias irracionales y prejuicios acerca de la homosexualidad, que hoy en día siguen afectando a nuestra sociedad, sobre todo a aquellas personas que se identifican como miembros del colectivo LGTBI. Como resultado, se ha establecido un estigma asociado a la homosexualidad, que sigue imperando en las personas con una orientación sexual alternativa a la heterosexual donde tiene un papel importante en el desarrollo personal a nivel psicológico y social (Bauermeister, Sandfort, Eisenberg, Grossman, \& D’Augelli, 2010; Morell, Capel, \& Gajardo, 2005). Este estigma social tiene especial relevancia en la fase de negación donde, tras la presión social, aparecen intentos de experimentar con personas de otro sexo u ocultar su verdadera orientación para sentir así la aceptación del entorno (Cornejo, 2015). Además, las personas pertenecientes a este colectivo se enfrentan a otras barreras de discriminación debido a su orientación sexual, tanto en el ámbito familiar como en el social, como son la pertenencia a una minoría sexual, reconocimiento de un estigma y la presunción de cisheteronormatividad (Castillo, 2010; Russell, Ryan, Toomey, Díaz, \& Sánchez, 2011).

Todo ello conlleva una serie de consecuencias asociadas a este tipo de discriminación Igtbifóbica, como son el estrés derivado de pertenecer a una minoría sexual, es decir el miedo al rechazo por una cualidad intrínseca de la persona, que no se puede modificar ni es el resultado de una libre elección (Cathal, 2017; Pulgarín, 2009), problemas relacionados con el establecimiento de lazos afectivos y diversos problemas de salud mental, como son síntomas depresivos, ansiedad, baja autoestima e incluso ideaciones suicidas (Capistrant \& Nakash, 2018; Marchueta \& Echebarria 2014). Todas estas consecuencias derivan en uno de los mayores problemas asociados a estas actitudes negativas hacia el colectivo LGTBI por parte de la sociedad, la homofobia interiorizada (Newcomb \& Mustanski, 2010). Esta es definida como la interiorización de los prejuicios negativos de la sociedad hacia las personas no heterosexuales y genera, en muchos casos, la no aceptación de la propia homosexualidad o una aceptación con restricciones, por ejemplo, la ocultación de la propia orientación sexual o su vivencia con elevada preocupación (Meyer, 2013).

Dada la gravedad de las repercusiones que puede tener la vivencia de una orientación sexual distinta a la heterosexual, el objetivo del presente estudio fue analizar la preocupación informada por la propia orientación sexual en un grupo de adolescentes y jóvenes heterosexuales y no heterosexuales, y la influencia de la edad de la toma de conciencia en esta preocupación. 


\section{PREOCUPACIÓN ASOCIADA AL DESCUBRIMIENTO DE LA ORIENTACIÓN SEXUAL}

\section{MÉTODO}

\section{Participantes}

Se evaluó a un total de 140 estudiantes de dos centros públicos de educación secundaria de la provincia de Castellón (España), 91 mujeres (65\%) y 49 hombres (35\%). Las edades del alumnado estaban comprendidas entre los 12 y los 25 años ( $M=15.66$; DT=3.88).

\section{Instrumentos de evaluación}

Las personas participantes cumplimentaron un cuestionario on-line desarrollado ad hoc sobre actitudes hacia el colectivo LGTBI en las aulas y conocimientos sobre conceptos de diversidad afectivo sexual y de género denominado "Cuestionario sobre Discriminación y Diversidad Sexual" (Salusex, 2018). Este instrumento recogía, por un lado, 4 variables sociodemográficas y, por el otro, 57 preguntas repartidas en bloques de vivencia y preocupación sobre la orientación sexual, conocimientos, actitudes, intención de conducta y de comportamiento sobre discriminación y diversidad sexual. Para este estudio se han seleccionado 4 ítems:

Un ítem que evalúa la orientación sexual "Sientes atracción hacia:" (hombres, mujeres, hombres y mujeres, nadie, no lo tengo claro).

Tres ítems que evalúan la vivencia y la preocupación por la orientación sexual: un primer ítem de respuesta abierta "¿A qué edad te diste cuenta de tu orientación sexual?"; y dos ítems con escala de respuesta tipo Likert (0 = nada, 1 = un poco, 2 = bastante y 3 = mucho) que evalúan la preocupación sobre la orientación sexual en el pasado ( ¿Sentiste preocupación o angustia al darte cuenta?") y en el presente ( "En la actualidad, ¿te preocupa o angustia tu orientación sexual?").

\section{Procedimiento}

Una vez obtenidos los permisos necesarios por parte de la Conselleria de Educación y de los centros educativos, se inició el proceso de recogida de datos de manera online. Esta recogida se produjo durante los meses de diciembre de 2018 y mayo de 2019 en los propios institutos, siendo las personas investigadoras del estudio quiénes se desplazaron hasta los centros educativos, siempre dentro del horario escolar, para informar del estudio al alumnado y profesorado. Una vez dado su consentimiento, Ios participantes accedían al cuestionario online, el cual se cumplimentaba en un promedio de 30 minutos. Después de contestar el cuestionario, recibían un taller de dos horas sobre diversidad afectivo-sexual y de género. La participación en el estudio fue totalmente anónima, confidencial y voluntaria.

\section{Análisis estadísticos}

Para realizar los diferentes análisis se utilizó el programa estadístico SPSS versión 25. En primer lugar, se utilizaron frecuencias para las variables categóricas (por ejemplo, género) y medias y desviaciones típicas para las variables cuantitativas (por ejemplo, edad) con el objetivo de describir las características sociodemográficas de la muestra. En segundo lugar, para examinar las diferencias en la preocupación por la orientación sexual pasada y presente entre grupos se utilizó la prueba Chi-cuadrado. Para ello, se categorizó a la muestra en dos grupos en función de la orientación sexual, excluyendo a las personas que se habían identificado como asexuales. Por Io tanto, el grupo de heterosexuales representaba el $76 \%(n=117)$ y el grupo de no heterosexuales representaba el $14,2 \%(n=23)$. En tercer lugar, se siguió utilizando la prueba Chi-cuadrado para examinar las diferencias en la preocupación por la orientación sexual presente en función de la edad. Para ello, se categorizó a la muestra en dos grupos etarios, uno de 12 a 18 años (adolescentes), que representaba el $67.2 \%(n=94)$ del total de la muestra, y otro de 19 a 25 años (jóvenes), que representaba el 32.8\% (n=46). Por último, para analizar la relación entre la edad a la que se dieron cuenta o tomaron conciencia de su orientación y el nivel de preocupación en la actualidad, se realizó una correlación de Spearman para cada orientación sexual por separado (heterosexual y no heterosexual). 


\section{RESULTADOS}

1. Frecuencia de orientación sexual informada y edad de toma de conciencia

En general, el $76 \%$ de la muestra se identificaba como heterosexual, el $4.5 \%$ como bisexual, el $6.5 \%$ como homosexual, el $9.7 \%$ como asexual y el $3.2 \%$ se estaba cuestionando su orientación sexual en el momento de la evaluación. Es decir, un $20.8 \%$ de los participantes se identificaba con una orientación sexual no heterosexual. La toma de conciencia de la orientación sexual fue posterior en las personas no heterosexuales ( $M=10.45$ años; $\mathrm{DT}=6.43)$ que en las heterosexuales ( $\mathrm{M}=5.74$ años; $\mathrm{DT}=5.04)$, mostrando diferencias estadísticamente significativas $(t=3.84 ; p £ .001)$.

En función de la edad, se observa un mayor porcentaje de personas que se identifican como heterosexuales tanto en adolescentes como en jóvenes, sin embargo las orientaciones no heterosexuales aumentan a partir de los 19 años. Por otro lado, se observa que en la adolescencia existe un 14\% que se identifican como asexuales, porcentaje que desaparece en el grupo de jóvenes. De igual modo, cabe destacar que el cuestionamiento y las dudas se reduce a la mitad en los jóvenes (véase Tabla 1).

Tabla 1. Frecuencia de orientación sexual por grupo etario

\begin{tabular}{lcc}
\hline & 12 a 18 años $(\mathrm{n}=94)$ & 19 a 25 años $(\mathrm{n}=46)$ \\
\hline Heterosexual & $\%$ & $\%$ \\
Bisexual & $76.6 \%$ & $71.1 \%$ \\
Homosexual & $1.1 \%$ & $13 \%$ \\
Cuestionamiento & $3 \%$ & $13 \%$ \\
Asexual & $4 \%$ & $2.2 \%$ \\
\hline
\end{tabular}

2. Relación entre la orientación sexual y el nivel de preocupación o angustia experimentada en el momento de toma de conciencia y en la actualidad

En primer lugar, al analizar el nivel de preocupación con la orientación sexual (véase Tabla 2), se observa que las personas no heterosexuales mostraron mayor preocupación que las personas heterosexuales al darse cuenta de su orientación en el pasado. De hecho, un $45.5 \%$ de personas no heterosexuales, frente a un $3.4 \%$ de personas heterosexuales, mostraron en algún grado preocupación con su orientación sexual. En segundo lugar, en cuanto a la preocupación en la actualidad, se sigue observando un mayor nivel de preocupación en el grupo de personas no heterosexuales que en el grupo de heterosexuales (31.8\% vs. 3.5\%). No obstante, cabe destacar que en las personas heterosexuales esta preocupación se mantiene similar a la obtenida en el pasado con un $3.5 \%$ de las personas mostrando algún grado de preocupación, mientras que disminuye en el caso de las personas no heterosexuales pasando de un $45.4 \%$ a un $31.8 \%$ y con menor intensidad en la preocupación, siendo estas diferencias significativas a nivel estadístico $\left(\chi^{2}=23.838 ; \mathrm{p} £ .001\right)$.

Tabla 2. Relación entre preocupación por la orientación sexual en el pasado y el presente y orientación sexual informada

\begin{tabular}{|c|c|c|c|c|c|c|}
\hline \multicolumn{4}{|c|}{ Al darse cuenta } & \multicolumn{3}{|c|}{ En la actualidad } \\
\hline & $\begin{array}{l}\text { Heterosexual } \\
\quad(\mathrm{n}=117)\end{array}$ & $\begin{array}{c}\text { No } \\
\text { heterosexual } \\
(\mathrm{n}=22)\end{array}$ & $\chi^{2}(p)$ & $\begin{array}{l}\text { Heterosexual } \\
\quad(\mathrm{n}=117)\end{array}$ & $\begin{array}{c}\text { No } \\
\text { heterosexual } \\
(\mathrm{n}=22)\end{array}$ & $\chi^{2}(p)$ \\
\hline Nada & $96.6 \%$ & $54.5 \%$ & & $96.5 \%$ & $68.2 \%$ & \\
\hline Un poco & $0.9 \%$ & $27.3 \%$ & $\chi^{2}=40.505$ & $2.6 \%$ & $31.8 \%$ & $\chi^{2}=23.838 ;$ \\
\hline Bastante & $0.9 \%$ & $13.6 \%$ & $\mathrm{p} \leq .001$ & $0 \%$ & $0 \%$ & $\mathrm{p} \leq .001$ \\
\hline Mucho & $1.7 \%$ & $4.5 \%$ & & $0.9 \%$ & $0 \%$ & \\
\hline
\end{tabular}


3. Diferencias en el nivel de preocupación o angustia experimentada en la actualidad con respecto a la orientación sexual en heterosexuales y no heterosexuales en función de la edad.

Pese a que no se encuentran diferencias estadísticamente significativas entre el nivel de preocupación actual por la orientación sexual y los dos grupos de edades en heterosexuales y en no heterosexuales (véase Tabla 3), cabe señalar que hay menor preocupación por la orientación sexual en el grupo de heterosexuales en cualquier rango de edad. Y, por otra parte, el mayor porcentaje de preocupación en el grupo de no heterosexuales aparece entre los 19 y 25 años.

Tabla 3. Relación entre preocupación por la orientación sexual en el presente y edad en función de la orientación sexual informada

\begin{tabular}{llccc}
\hline & \multicolumn{1}{c}{12 a 18 años } & 19 a 25 años & $\chi^{2}(\mathrm{p})$ \\
\hline Heterosexuales & Nada & $95.8 \%$ & $97 \%$ & \\
$(\mathrm{n}=117)$ & Un poco & $2.8 \%$ & $3 \%$ & $\chi^{2}=0.466 ; \mathrm{p}=.792$ \\
& Bastante & $0 \%$ & $0 \%$ & \\
& Mucho & $1.4 \%$ & $0 \%$ & \\
No & Nada & $75 \%$ & $61.5 \%$ & \\
heterosexuales & Un poco & $25 \%$ & $38.5 \%$ & $\chi^{2}=0.404 ; \mathrm{p}=.525$ \\
$(\mathrm{n}=22)$ & Bastante & $0 \%$ & $0 \%$ & \\
& Mucho & $0 \%$ & $0 \%$ & \\
\hline
\end{tabular}

Relación entre edad de toma de conciencia y el nivel de preocupación o angustia experimentada en el pasado y en la actualidad con respecto a la orientación sexual en heterosexuales y no heterosexuales

Por último, las correlaciones entre la edad a la que tomaron conciencia de su orientación sexual y el nivel de preocupación pasado y presente resultan estadísticamente significativas solo en la población no heterosexual respecto a la preocupación experimentada en la actualidad. Es decir, cuanto más tarde se dieron cuenta de su orientación sexual, mayores niveles de preocupación informan tener en la actualidad ( $R h 0=0.432 ; p=.045$ ) (véase Tabla 4).

Tabla 4. Correlación entre la edad de la toma de conciencia de la orientación sexual y preocupación pasada y presente en heterosexuales y no heterosexuales

\begin{tabular}{|c|c|c|}
\hline & & $\begin{array}{l}\text { ¿A qué edad te diste cuenta de tu orientación } \\
\text { sexual? }\end{array}$ \\
\hline \multirow{2}{*}{$\begin{array}{l}\text { Preocupación al } \\
\text { darse cuenta }\end{array}$} & Heterosexual & Rho $=-0.009 ; p=.925$ \\
\hline & No heterosexual & $\mathrm{Rho}=0.431 ; \mathrm{p}=.065$ \\
\hline \multirow{2}{*}{$\begin{array}{l}\text { Preocupación en } \\
\text { la actualidad }\end{array}$} & Heterosexual & Rho $=-0.121 ; p=.194$ \\
\hline & No heterosexual & $\mathrm{Rho}=0.432 ; \mathrm{p}=.045$ \\
\hline
\end{tabular}

\section{DISCUSIÓN}

Construir un proceso identitario adecuado, sano y libre de prejuicios es de vital importancia para no desarrollar problemas de salud mental y/o problemas en la construcción de lazos afectivos. Son varios los factores que influyen en este proceso identitario como son la edad, la etapa evolutiva en que se toma conciencia de la homosexualidad, la mayor o menor adecuación a los roles de género y a las normas del entorno. Para este estudio nos hemos centrado en la importancia de la etapa evolutiva de la toma de conciencia de la orientación de los jóvenes y su relación con el desarrollo de los procesos identitarios en la adolescencia. Como aspecto diferencial a otros estudios, hemos realizado comparativas entre personas heterosexuales vs no heterosexuales para hacer visible la realidad de las personas jóvenes del colectivo LGTBI. 
En términos generales, en el proceso identitario de la orientación sexual, observamos que en las edades entre 19 y 25 años, el número de personas que se identifican como heterosexuales descienden. Así mismo vemos cómo también desciende el número de personas que están en cuestionamiento y un descenso total de las personas asexuales; y a su vez aumenta el número de bisexuales y homosexuales. Pese a que estos datos no son longitudinales, sino transversales, están en la línea del estudio de Koswic, Palmer \& Kull (2015), indicando una posible tendencia según se construye la orientación sexual a que esta sea más diversa en edades más avanzadas y, por lo tanto, menos heteronormativa. Estos resultados pueden estar en relación a otros estudios como el de Bardi, Leyton, Martínez \& González (2005) que indican que la adquisición de la orientación no heterosexual es un largo proceso que empieza con el reconocimiento de sentimientos homosexuales e inicia su proceso de aceptación una vez se establecen las primeras experiencias afectivo-sexuales. Por otro lado, el hecho de que el porcentaje de asexuales baje del 14\% antes de los 19 años a 0\% nos habla de la existencia de una asexualidad evolutiva y de cómo la orientación sexual es algo que se puede desarrollar a lo largo del ciclo vital, lo que no significa que con posterioridad a esa edad no podamos encontrar a personas que siguen sin sentir atracción hacia ningún sexo.

Al analizar con más detenimiento el proceso identitario, observamos que la preocupación mostrada en el momento de toma de conciencia de la propia orientación sexual es diferente en función de la orientación. Las personas no heterosexuales muestran mayor preocupación; esto puede deberse, tal y como defienden Kertzner, Meyer, Frost \& Stirratt (2009), a la influencia del contexto social y la presión de pertenecer a un grupo sexual minoritario. A todo ello se le suma el marco heteronormativo de la sociedad, el cual hace que el hecho de manifestar atracción sexual hacia personas del mismo sexo genere malestar. Cuando analizamos esta preocupación en la actualidad, observamos que esta disminuye para todas las personas, aunque en las personas no heterosexuales esta preocupación sigue siendo elevada. Esta preocupación puede ir ligada a un rechazo hacia la propia homosexualidad (homofobia interiorizada) tal y como defiende Castro (2010). Estos datos coinciden con el proceso identitario propuesto por Soriano (2004) quien remarca que la aceptación de la orientación sexual no heterosexual es un proceso que puede durar años y que se encuentra en constante interacción del entorno y este puede influenciar retrasando o facilitando el proceso.

Más concretamente, al analizar estas vivencias de las personas no heterosexuales en función de la edad, vemos que las personas no heterosexuales de edades entre 12 y 18 años presentan menor malestar que las de mayor edad. Esto puede deberse, en línea de los hallazgos encontrados por Cornejo (2015), a que pertenecen a una generación en la que existe una mayor normalización de la diversidad sexual en la sociedad. Este aumento de la visibilización de la diversidad hace que las personas menores hayan crecido en un contexto de menor homofobia que las de 19 a 25 años. No obstante habría que considerar la posibilidad de realizar un estudio Iongitudinal 0 ampliar las edades la muestra para así poder explorar con mayor exactitud esta posibilidad.

Una de las variables que pueden influir en este malestar es la edad en la que surge la toma de conciencia de la propia orientación. Los resultados muestran que las personas heterosexuales, señalan como media poco antes de los 6 años de edad, mientras que las personas no heterosexuales alrededor de los 10 años. Sin embargo estudios como el de Soriano (2004) defienden que la edad de toma de conciencia de la orientación sexual se establece alrededor de los 10 a 14 años, estando en las personas heterosexuales alrededor de los 12 años y de las no heterosexuales alrededor de los 11 años. Este hecho nos hace pensar que las diferencias encontradas en relación a la edad en la que se descubre la orientación, pueden estar influidas por el sesgo de interpretación de creer que se nace con una orientación determinada y no con el hecho de que se trata de una construcción social a lo largo de una etapa como defiende Soriano (2004). El proceso identitario y de desarrollo de la orientación está formado por una serie de fases según cada momento evolutivo, tal y como explica Cass (1979) en su Modelo de formación de la identidad homosexual y, en este sentido, a edades tan tempranas no se dispone de suficiente capacidad introspectiva para tomar conciencia de la propia orientación. Con todo ello, al analizar la posible relación entre edad y malestar por la orientación, se observa que las personas no heterosexuales que presentan mayor malestar 
en la actualidad son aquellas que tomaron conciencia de su orientación más tarde. Esto puede estar relacionado al hecho de que el proceso de aceptación de la orientación está relacionado con el momento vital de la persona y a la fase en la que se encuentre dentro del proceso identitario, y no tanto a la edad que tengan las personas. Dube (2000) establece que el proceso de aceptación se da en las últimas partes del proceso, donde generalmente la persona revela su orientación a su entorno y de ese modo empieza a desarrollar una actitud positiva hacia la propia orientación sexual.

Con toda la información obtenida en este estudio, consideramos que para futuras investigaciones sería interesante aumentar el número de participantes, de forma que se pudieran obtener resultados más concluyentes y permitiera una mayor exploración. Así mismo, plantearnos encontrar una muestra más equitativa, donde se equiparará en número de hombres y mujeres en cada una de las orientaciones planteadas, para así poder realizar diferencias por género y ver si estas influyen en los resultados obtenidos como muestran otros estudios como el de Peterseny y Hyde (2011). Así como explorar otras variables psicosociales que puedan estar influyendo en dichas diferencias.

Estos resultados muestran que todavía jóvenes y adolescentes presentan preocupación sobre su propia orientación sexual, sobre todo cuando la identificación como no heterosexual se hace a una edad más tardía. Queda patente la importancia de normalizar la diversidad afectivo-sexual, y la necesidad de romper con la heteronormatividad, para que identificarse como no heterosexual no implique ningún tipo de preocupación que pueda ser la antesala de problemas de salud mental. Por ello desde la prevención consideramos de vital importancia detectar y eliminar aquellas actitudes y conductas homofóbicas, así como leyes que priven de derechos al colectivo LGTBI. Se trata de una responsabilidad de toda la sociedad el establecer modelos de relaciones donde se fomenten la diversidad y la libertad por igual independientemente de la orientación afectivo-sexual y de género. Por ese motivo desde la educación se llevan acabo acciones de sensibilización y formación inclusivas en las aulas, con perspectiva LGTBI, para así construir sociedades más libres y tolerantes.

\section{REFERENCIAS BIBLIOGRÁFICAS}

Asamblea general de la asociación mundial de sexología (Junio, 1997). Declaración universal de los derechos sexuales. Comunicación presentada en el XIII Congreso Mundial de Sexología. Valencia.

Bardi, A., Leyton, C., Martínez, V., \& González, E. (2005). Identidad Sexual: proceso de definición en la adolescencia. Reflexiones Pedagógicas, 26, 43-51.

Bauermeister, J., Sandfort, T., Eisenberg, A., Grossman, A., \& D’Augelli, A. (2010). Relationship trajectories and psychological well-being among sexual minority youth. Journal of Youth and Adolescence, 39(10), 11481163.

Cathal, S. (2017). El impacto humano de la ley de "propaganda gay" de Rusia. IFEX La red global: Defendiendo y promoviendo la libertad de expresión. Disponible en https://www.ifex.org/russia/2017/03/02/gay-propaganda-law/es/

Cass, V. C. (1979). Homosexuality identity formation: A theoretical model. Journal of Homosexuality, 4(3), 219235.

Capistrant, B. D., \& Nakash, 0. (2019). Suicide risk for sexual minorities in middle and older age: evidence from the National Survey on Drug Use and Health. The American Journal of Geriatric Psychiatry, 27(5), 559-563.

Cornejo, J. (2015). Componentes ideológicos de la homofobia. Límite. Revista de Filosofía y Psicología, 7(26), 85-106.

Dube, E. M. (2000). The role of sexual behavior in the identification process of gay and bisexual males. Journal of Sex Research, 37(2), 123-132.

Kertzner, R., Meyer, H., Frost M., \& Stirratt, M. (2009). Social and psychological well- being in Lesbians, Gay Men, and Bisexuals: The effects of race, gender, age, and sexual identity. American Journal of Orthopsychiatry, 79(4), 500-510. 
Kosciw, G., Neal A., \& Ryan M. (2015). Reflecting resiliency: openness about sexual orientation and/or gender identity and its relationship to well-being and educational outcomes for LGBT students. American Journal of Community Psychology, 55(1-2): 167-178.

Herz, M. \& Johansson, T. (2015). The normativity of the concept of heteronormativity. Journal of Homosexuality, 62(8), 1009-1020.

Martxueta, A. \& Etxeberria, J. (2014). Análisis diferencial retrospectivo de las variables de salud mental en lesbianas, gais y bisexuales (LGB) víctimas de bullying homofóbico en la escuela. Revista de Psicopatología y Psicología Clínica, 19(1), 23-35.

Meyer, I. (2013). Prejudice, social stress, and mental health in lesbian, gay, and bisexual populations: Conceptual issues and research evidence. Psychology of Sexual Orientation and Gender Diversity, 1, 3-26.

Montagut, E. (2015). Historia de España: El franquismo contra los homosexuales. Nuevatribuna.es (on line). Disponible en http://www.nuevatribuna.es/articulo/cultura-ocio/franquismo- homosexuales/20150810185411118973.html

Morell, S. \& Gajardo, D. (2005, noviembre). Consecuencias psicologicas del Bullying homofobico y transfobico: factores identificativos y preventivos. Articulo presentado en forma de taller en el IV Seminario de la AMPGIL (Associacio de mares, pares de gays i lesbianes) Barcelona, Espana.

Newcomb, Michael E., \& Brian Mustanski. (2010). Internalized homophobia and internalizing mental health probIems: A meta-analytic review. Clinical Psychology Review, 30(8), 1019-1029.

Pulgarín, J. M. P. (2009). Entre la discriminación y el reconocimiento: las minorías sexuales en materia de educación. Revista Vía luris, 7, 29-41.

Petersen, J. L. \& Hyde, J. S. (2011). Gender differences in sexual attitudes and behaviors: A review of meta-anaIytic results and large datasets. Journal of Sex Research, 48(2-3), 149-165.

Russell, S. T., Ryan, C., Toomey, R. B., Diaz, R. M. \& Sanchez, J. (2011). Lesbian, gay, bisexual, and transgender adolescent school victimization: Implications for young adult health and adjustment. Journal of School Health, 81(5), 223-230.

Soriano, S. (2004). Cómo se vive la homosexualidad y el lesbianismo (2ª edición). Salamanca: Amarú Ediciones. 
